\title{
Urticaria vasculítica en un paciente con glioblastoma tratado con temozolamida y levetiracetam
}

\author{
Urticarial vasculitis in a patient with glioblastoma treated with temozolomide \\ and levetiracetam
}

\section{Andrés Quintero ${ }^{1,2}$, Andrés Felipe Cardona2-4, Luisa Neira ${ }^{5}$, Laura Bernal ${ }^{6}$}

'Departamento de Neurocirugía, Fundación Santa Fe de Bogotá (Bogotá, Colombia).

Instituto de Neurociencias, Universidad El Bosque (Bogotá, Colombia).

${ }^{3}$ Grupo de Oncologí Clínica y Traslacional Instituto de Oncología Fundación Santa Fe de Bogotá (Bogotá Colombia).

4Fundación para la Investigación Clinica y Molecular Aplicada del Cáncer (FICMAC): investigador asociado ONCOLGroup.

Grupo Oncología Clínica Cansercoop (Bogotá, Colombia).

${ }^{6}$ Sección Investigación Clínica y Traslacional, Instituto de Oncología, Fundación Santa Fe de Bogotá (Bogotá, Colombia).

Se presentan los hallazgos clínicos de una mujer de 26 años con un glioblastoma primario tratado inicialmente con una resección quirúrgica subóptima (evaluación volumétrica cercana al 90\%). La patología reveló una neoplasia con un $\mathrm{Kl}_{67}$ del 40\% portadora de un fenotipo metilador por la alteración del promotor de la MGMT (O-6-metilguanin-ADN metiltransferasa) y positividad para la mutación R132H de la IDH1 (Isocitrato deshidrogenasa 1). Inició tratamiento siguiendo la plataforma propuesta por Stupp y colaboradores' completando 18 meses del alquilante sin progresión de la enfermedad. Siete meses después de la supresión del tratamiento médico, se documentó compromiso focal por la enfermedad que derivó en una paresia densa del hemicuerpo izquierdo y en una epilepsia sintomática secundaria. Se llevó a cabo una nueva resección seguida de reirradiación (pauta hipofraccionada), bevacizumab (10 mg/kg) y temozolamida metronómica ( 75 mg/m²/día por 21 días cada 28 días); como intervención adicional, se dejó levetiracetam 500 mg cada 12 horas.

Luego de completar tres meses de manejo con mínima toxicidad y recuperación funcional parcial (Barthel $80 \%)$, se encontraron múltiples lesiones maculares eritematosas y pruriginosas que seguían un patrón urticariforme; considerando los hallazgos se debieron a la administración de alguno de los medicamentos, se suprimió de forma secuencial la temozolamida y el antiepiléptico, logrando una disminución progresiva de las alteraciones después de introducir el uso de esteroides. La figura 1 muestra la extensa erupción eritematosa que afectaba el cuello, el dorso y los miembros inferiores. En la figura 2, se observa el hallazgo de las biopsias por punción donde se evidenció una extensa infiltración dérmica compuesta por neutrófilos, eosinófilos y linfocitos.
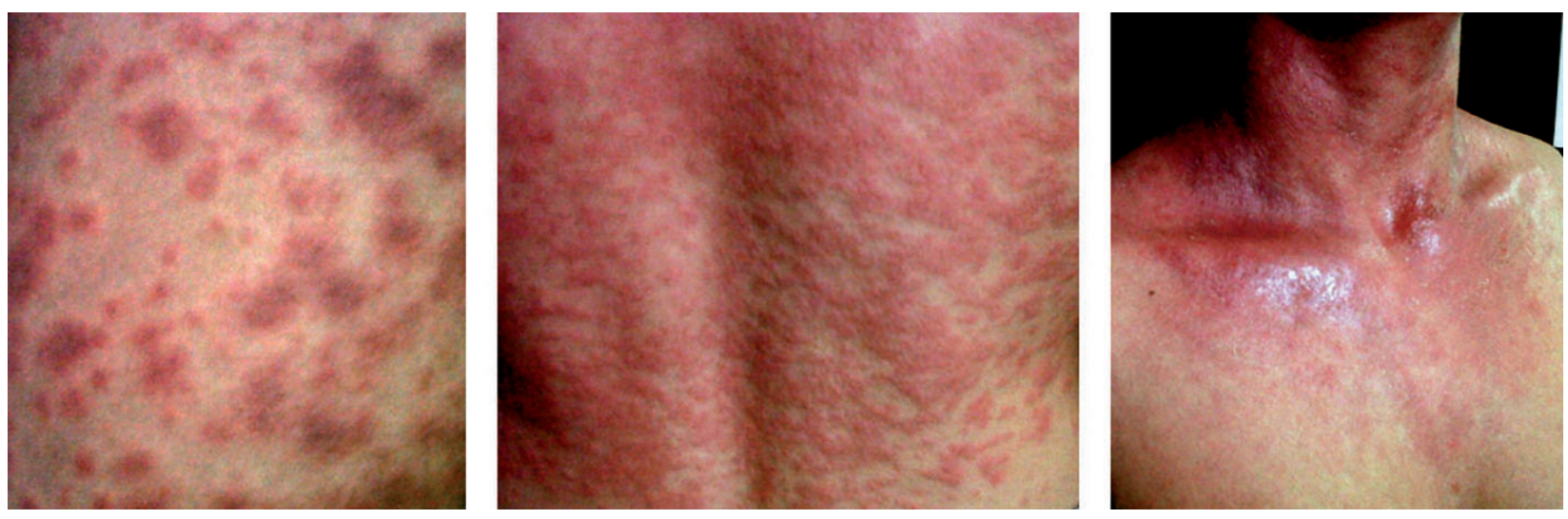

Figura 1. Lesiones de patrón urticariforme que afectaron la cara, el cuello, el dorso y los miembros inferiores. 


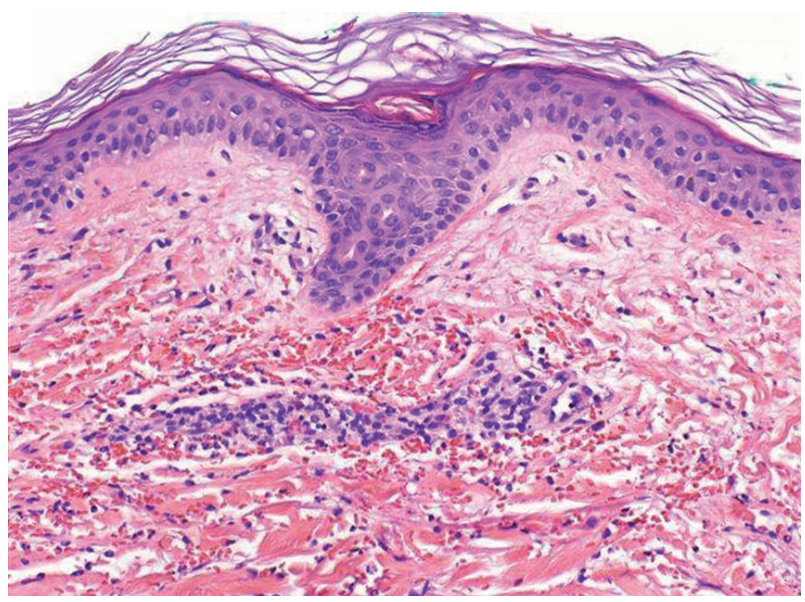

Figura 2. Extenso infiltrado linfocitario y esonofilico perivascular.

La urticaria vasculítica consiste en lesiones cutáneas eritematosas de apariencia urticariforme con una duración mayor a 24 horas en relación con cambios inflamatorios perivasculares en el examen microscópico. Estos casos se han asociado a otras patologías sistémicas de origen reumatológico, como el lupus eritematoso, la artritis y el síndrome de Sjögren. Posteriormente, William y colaboradores la describieron en asociación con algunos antibióticos, antihipertensivos y diuréticos ${ }^{2}$. El diagnóstico inicial es clínico y se confirma con el análisis del tejido ${ }^{3}$. El tratamiento depende de la causa subyacente y se sugiere la medición regular de los niveles de complemento en sangre para definir el pronóstico, siendo peor en presencia de manifestaciones más severas, incluyendo la afectación visceral en pulmón y riñón ${ }^{4}$.

La temozolamida es un agente alquilante, utilizado ampliamente en pacientes con gliomas de alto grado, para el manejo del melanoma y de los tumores neuroendocrinos. Los efectos adversos más significativos son la trombocitopenia, leucopenia, fatiga, náuseas y la neumonía inducida por Pneumocystis jiroveci. Según lo reportado por un estudio que recopiló 2658 pacientes que recibieron temozolamida, la presentación de

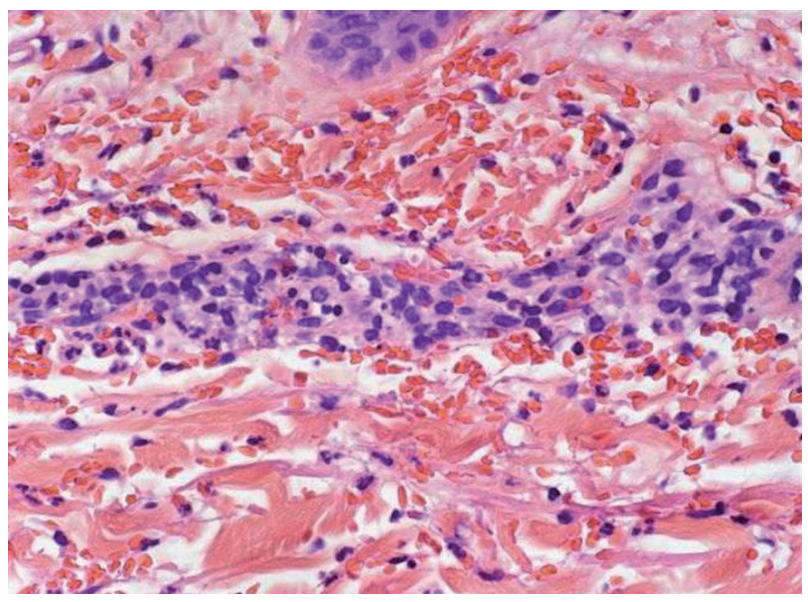

urticaria es rara $(0,08 \%)$, siendo más frecuente en las mujeres mayores de 60 años y durante la exposición a coadyuvantes, como los antiepilépticos ${ }^{5}$. De acuerdo con nuestro conocimiento, solo hay un reporte previo de hipersensibilidad urticarial en un paciente tratado con temozolamida y levetiracetam ${ }^{6}$. La importancia de este caso radica en el tiempo previo de exposición a la medicación y en la morbilidad que derivó en la supresión del alquilante y del anteiepilépico.

\section{Referencias}

1. Stupp R, Mason WP, van den Bent MJ, Weller M, Fisher B, Taphoorn MJ, et al. Radiotherapy plus concomitant and adjuvant temozolomide for glioblastoma. N Engl J Med. 2005;352(10):987-96.

2. James $W D$, Berger $T G$, et al. Andrews' diseases of the skin: clinical dermatology Saunders Elsevier. Postgrad Med J. 1990;66(781):984.

3. Wisnieski JJ. Urticarial vasculitis. Curr Opin Rheumatol. 2000;12(1):24-31.

4. Venzor J, Lee WL, Huston DP. Urticarial vasculitis. Clin Rev Allergy Immunol. 2002;23(2):201-16.

5. Temozolomide and urticaria. Disponible en: <http://www. ehealthme.com>.

6. Pothiawala S, Hsu MY, Yang C, Kesari S, Ibrahimi OA. Urticarial hypersensitivity reaction caused by temozolomide. J Drugs Dermatol. 2010 Sep;9(9):1142-4. 